\title{
Exact exponential-time algorithms for finding bicliques
}

\author{
Daniel Binkele-Raible ${ }^{\mathrm{a}}$, Henning Fernau ${ }^{\mathrm{a}}$, Serge Gaspers ${ }^{\mathrm{b}}$, Mathieu Liedloff ${ }^{\mathrm{c}, *}$ \\ ${ }^{a}$ Universität Trier, FB 4-Abteilung Informatik, D-54286 Trier, Germany \\ ${ }^{b}$ CMM, Universidad de Chile, Av. Blanco Encalada 2120, Santiago de Chile \\ ${ }^{c}$ LIFO, Université d'Orléans, 45067 Orléans Cedex 2, France
}

\begin{abstract}
Due to a large number of applications, bicliques of graphs have been widely considered in the literature. This paper focuses on non-induced bicliques. Given a graph $G=(V, E)$ on $n$ vertices, a pair $(X, Y)$, with $X, Y \subseteq V, X \cap Y=\emptyset$, is a non-induced biclique if $\{x, y\} \in E$ for any $x \in X$ and $y \in Y$. The NP-complete problem of finding a non-induced $\left(k_{1}, k_{2}\right)$-biclique asks to decide whether $G$ contains a non-induced biclique $(X, Y)$ such that $|X|=k_{1}$ and $|Y|=k_{2}$. In this paper, we design a polynomial-space $\mathcal{O}\left(1.6914^{n}\right)$ time algorithm for this problem. It is based on an algorithm for bipartite graphs that runs in time $\mathcal{O}\left(1.30052^{n}\right)$. In deriving this algorithm, we also exhibit a relation to the spare allocation problem known from memory chip fabrication.
\end{abstract}

Key words: exact exponential-time algorithms, NP-hard problem, complete bipartite subgraphs

\section{Introduction}

Throughout the paper all graphs $G=(V, E)$ are undirected and simple. An induced biclique of $G$ is a complete bipartite induced subgraph of $G$. A non-induced biclique is a complete bipartite (not necessarily induced) subgraph of $G$. Equivalently, the pair $(X, Y)$ of disjoint vertex subsets $X \subseteq V$ and $Y \subseteq V$ is a non-induced biclique of $G$ if $\{x, y\} \in E$ for all $x \in X$ and $y \in Y$. If, additionally, $X$ and $Y$ are independent sets, then $(X, Y)$ is an induced biclique of $G$. Notice that, if $G$ is a bipartite graph, then every non-induced biclique of $G$ is also an induced one. Let the pair $(X, Y)$ be an induced or non-induced biclique of $G$. Then we call it a $\left(k_{1}, k_{2}\right)$ biclique if $|X|=k_{1}$ and $|Y|=k_{2}$. Its cardinality is $|X|+|Y|$.

The literature dealing with bicliques is rich and diverse. There are applications of bicliques (induced or non-induced on general or bipartite graphs) in various different areas such as data mining, automata and language theory, artificial intelligence and biology; see for example [1]. Therefore, bicliques and algorithmic problems about bicliques have been studied extensively.

\footnotetext{
* Corresponding author

Email addresses: raible@informatik.uni-trier.de (Daniel Binkele-Raible), fernau@uni-trier.de (Henning Fernau), sgaspers@im.uchile.cl (Serge Gaspers), liedloff@univ-orleans.fr (Mathieu Liedloff)

Preprint submitted to Elsevier
} 
Known results. Already in [8], the complexity of finding certain bicliques has been considered. For example, deciding whether a bipartite graph has a $(k, k)$ biclique, also known as a balanced biclique of size (at least) $k$, is NP-complete ([GT24] in [8]). A maximum cardinality induced biclique can be computed in polynomial time on bipartite graphs [3], whereas this problem is NP-complete for general graphs [15]. A related problem that asks to compute a non-induced biclique with a maximum number of edges is also known to be NP-hard [14]. Studies on the approximability of these problems are presented in [11].

The above-mentioned NP-completeness of the balanced biclique problem on bipartite graphs implies the NP-completeness of the following three problems about the existence of induced and non-induced bicliques, respectively.

\section{Induced $\left(\mathbf{k}_{\mathbf{1}}, \mathbf{k}_{\mathbf{2}}\right)$ Biclique}

Input: An undirected graph $G$, positive integers $k_{1}$ and $k_{2}$.

Question: Does $G$ have an induced $\left(k_{1}, k_{2}\right)$ biclique?

\section{Non-Induced $\left(\mathbf{k}_{1}, \mathbf{k}_{2}\right)$ Biclique}

Input: An undirected graph $G$, positive integers $k_{1}$ and $k_{2}$.

Question: Does $G$ have a non-induced $\left(k_{1}, k_{2}\right)$ biclique?

\section{Bipartite $\left(\mathbf{k}_{1}, \mathbf{k}_{2}\right)$ Biclique}

Input: An undirected bipartite graph $G$, positive integers $k_{1}$ and $k_{2}$.

Question: Does $G$ have a $\left(k_{1}, k_{2}\right)$ biclique?

Observe that any biclique in a bipartite graph as defined in the last problem definition is induced.

There is a trivial $\mathcal{O}^{*}\left(3^{n}\right)$ algorithm for finding and also for enumerating all induced and non-induced $\left(k_{1}, k_{2}\right)$ bicliques of a graph, respectively. ${ }^{1}$ It considers all partitions of the vertex set into $X, Y$ and $V \backslash(X \cup Y)$ and verifies for each whether $(X, Y)$ fulfills all conditions.

Our results. For enumerating all non-induced $\left(k_{1}, k_{2}\right)$ bicliques, note that there is no hope in obtaining a faster algorithm than the above-described $\mathcal{O}^{*}\left(3^{n}\right)$ algorithm, as a complete graph on $n$ vertices has $\Theta^{*}\left(3^{n}\right)$ non-induced $(\lfloor n / 3\rfloor,\lfloor n / 3\rfloor)$ bicliques. For solving the Non-Induced $\left(\mathbf{k}_{\mathbf{1}}, \mathbf{k}_{\mathbf{2}}\right)$ Biclique problem, however, we give a polynomial-space $\mathcal{O}\left(1.6914^{n}\right)$ time algorithm, based on a polynomial-space $\mathcal{O}\left(1.30052^{n}\right)$ time algorithm for Bipartite $\left(\mathbf{k}_{\mathbf{1}}, \mathbf{k}_{\mathbf{2}}\right)$ Biclique. That algorithm in turn employs a relation to a specific application, namely to Spare Allocation, which is inspired by memory chip fabrication. This relation may be interesting on its own.

Observe that there is also an $\mathcal{O}^{*}\left(3^{n / 3}\right)=\mathcal{O}\left(1.4423^{n}\right)$ time algorithm to solve Induced $\left(\mathbf{k}_{1}, \mathbf{k}_{\mathbf{2}}\right)$ Biclique. This algorithm is based on enumerating all maximal induced bicliques of the graph with a polynomial delay algorithm [5] and on the fact that an $n$-vertex graph has $\mathcal{O}^{*}\left(3^{n / 3}\right)$ maximal induced bicliques [10].

In this note we improve on algorithms presented in [6], where the following results were presented: (1) a polynomial-space $\mathcal{O}\left(1.8899^{n}\right)$ time algorithm and (2) an exponentialspace $\mathcal{O}\left(1.8458^{n}\right)$ time algorithm for solving the Non-Induced $\left(\mathbf{k}_{\mathbf{1}}, \mathbf{k}_{\mathbf{2}}\right)$ Biclique problem. Our new results make use of connections to a problem called Constraint Bipartite

\footnotetext{
${ }^{1}$ Throughout the paper we write $f(n)=\mathcal{O}^{*}(g(n))$ if $f(n) \leq p(n) \cdot g(n)$ for some polynomial $p(n)$.
} 
Vertex Cover for which a sophisticated branching algorithm was described in $[2,7]$ that has been analyzed from the viewpoint of parameterized complexity.

\section{Finding bicliques in bipartite graphs}

As we will exhibit, the Bipartite $\left(\mathbf{k}_{\mathbf{1}}, \mathbf{k}_{\mathbf{2}}\right)$ Biclique problem is closely related to the following problem that comes up (with certain variants till today) in the fabrication process of memory elements.

An instance of Spare Allocation (SAP) is given by a $n_{1} \times n_{2}$ binary matrix $A$ representing an erroneous chip with $A[r, c]=1$ if and only if the chip is faulty at position $[r, c]$, and the parameter(s), positive integers $k_{1}$ and $k_{2}$. The task is: Is there a reconfiguration strategy that repairs all faults and uses at most $k_{1}$ spare rows and at most $k_{2}$ spare columns?

With reconfiguration strategy, we mean a prescription which rows and columns from $A$ have to be replaced by spares. Kuo and Fuchs [12] provide a fundamental study of that problem. A review on the according literature is given in [2]. Put concisely, the "most widely used approach to reconfigurable VLSI" uses spare rows and columns to tolerate failures in rectangular arrays of identical computational elements, which may be as simple as memory cells or as complex as processor units. If a faulty cell is detected, the entire row or column is replaced by a spare one.

The following graph-theoretic problem can easily be seen to be equivalent to the previous problem via the adjacency matrix of a bipartite graph:

An instance of Constraint Bipartite Vertex Cover (CBVC) is given by a bipartite graph $G=\left(V_{1}, V_{2}, E\right)$, and the parameter(s), positive integers $k_{1}$ and $k_{2}$. The task is: Is there a vertex cover $C \subseteq V_{1} \cup V_{2}$ with $\left|C \cap V_{i}\right| \leq k_{i}$ for $i=1,2$ ?

It is known [7] that CBVC admits a quadratic problem kernel and a search tree algorithm with running time $1.3999^{k} n^{\mathcal{O}(1)}$, where $k=k_{1}+k_{2}$.

Let us call a valid solution of a CBVC instance a $\left(k_{1}, k_{2}\right)$ vertex cover.

Parameterized duality is usually defined by reparameterizing, say a vertex-selection problem on graphs, by considering $n-k$ instead of $k$ as the parameter, where $n$ is the number of vertices in the graph and $k$ is the solution size. For example, thanks to Gallai's identity, the parameterized dual of Vertex Cover is Independent Set. For vertex-selection problems on bipartite graphs, where we face two parameters $k_{1}, k_{2}$ corresponding to the number of vertices in each part of the vertex bipartition $\left(V_{1}, V_{2}\right)$ that are in the solution, it is natural to consider the reparameterization given by $\left(n_{1}-\right.$ $\left.k_{1}, n_{2}-k_{2}\right)$, where $n_{i}=\left|V_{i}\right|$, as the dual parameterization. Considering $k=k_{1}+k_{2}$ and $n=n_{1}+n_{2}$, one can see that this definition corresponds to the widely used notion of parameterized duality for one-parametric problems.

The bipartite complement of a bipartite graph $G=\left(V_{1}, V_{2}, E\right)$ is the bipartite graph $G^{C}=\left(V_{1}, V_{2}, E^{C}\right)$, where $E^{C}$ contains all edges between $V_{1}$-vertices and $V_{2}$-vertices that are not contained in $E$.

The following lemma formalizes that Spare Allocation can be solved in $c^{k_{1}+k_{2}} n^{\mathcal{O}(1)}$ time if and only if the parameterized dual of Bipartite $\left(\mathbf{k}_{\mathbf{1}}, \mathbf{k}_{\mathbf{2}}\right)$ Biclique can be solved in $c^{k_{1}+k_{2}} n^{\mathcal{O}(1)}$ time.

Lemma 1. The Spare Allocation problem is polynomially equivalent to the parameterized dual of Bipartite $\left(\mathbf{k}_{\mathbf{1}}, \mathbf{k}_{\mathbf{2}}\right)$ Biclique. 
Proof. As noticed above, we can consider a CBVC instance to start with, that is, a bipartite graph $G=\left(V_{1}, V_{2}, E\right)$, together with parameters $k_{1}, k_{2}$. The parameterized dual asks to find an independent set $I_{1} \cup I_{2}$ in $G$ with $I_{i} \subseteq V_{i}$ and $\left|I_{i}\right| \geq k_{i}^{\prime}=\left|V_{i}\right|-k_{i}$. Now, $C_{1} \cup C_{2}$ is a $\left(k_{1}, k_{2}\right)$ vertex cover of $G$ if and only if $\left(V_{1} \backslash C_{1}\right) \cup\left(V_{2} \backslash C_{2}\right)$ is an independent set in $G$ with $\left|V_{1}\right|-k_{1}$ vertices in $V_{1}$ and $\left|V_{2}\right|-k_{2}$ vertices in $V_{2}$ if and only if $\left(V_{1} \backslash C_{1}\right) \cup\left(V_{2} \backslash C_{2}\right)$ is a $\left(\left|V_{1}\right|-k_{1},\left|V_{2}\right|-k_{2}\right)$ biclique of the bipartite complement of $G$.

Clearly, the "trivial barrier" for moderately exponential-time algorithms for CBVC is $\mathcal{O}^{*}\left(2^{n / 2}\right)$ rather than $\mathcal{O}^{*}\left(2^{n}\right)$, since it is enough to consider all subsets of the smaller set of $V_{1}$ and $V_{2}$. Our result is the first exact algorithm for Constraint Bipartite Vertex Cover that breaks the trivial $\Theta^{*}\left(2^{n / 2}\right)$-barrier.

Theorem 2. Constraint Bipartite Vertex Cover and Bipartite $\left(\mathbf{k}_{\mathbf{1}}, \mathbf{k}_{\mathbf{2}}\right)$ Biclique can be solved in time $\mathcal{O}\left(1.30052^{n}\right)$, using polynomial space.

Proof. Let $G=\left(V_{1}, V_{2}, E\right)$ be an instance for Bipartite $\left(\mathbf{k}_{\mathbf{1}}, \mathbf{k}_{\mathbf{2}}\right)$ Biclique. Let $n_{1}=$ $\left|V_{1}\right|, n_{2}=\left|V_{2}\right|$, and $\alpha:=0.2189$. We will consider two algorithmic possibilities depending on $k_{1}+k_{2}$.

1. First, suppose $k_{1}+k_{2} \geq \alpha n$. According to Lemma 1 the problem of finding a $\left(k_{1}, k_{2}\right)$ biclique is equivalent to finding a $\left(k_{1}^{\prime}, k_{2}^{\prime}\right)$ vertex cover in the bipartite complement with $k_{1}^{\prime}=n_{1}-k_{1}$ and $k_{2}^{\prime}=n_{2}-k_{2}$. The algorithm in [7] solves CBVC in time $\mathcal{O}\left(1.3999^{k_{1}^{\prime}+k_{2}^{\prime}}\right)=\mathcal{O}\left(1.3999^{n-\left(k_{1}+k_{2}\right)}\right)$. As $k_{1}+k_{2} \geq \alpha n$ the running time is $\mathcal{O}\left(1.3999^{(1-\alpha) n}\right)=\mathcal{O}\left(1.30052^{n}\right)$.

2. Now, suppose $k_{1}+k_{2}<\alpha n$. Depending on the values of $k_{1}, k_{2}, n_{1}$, and $n_{2}$, the algorithm either enumerates all subsets $V_{1}^{\prime} \subseteq V_{1}$ of size $k_{1}$ and checks whether $\left|\bigcap_{v \in V_{1}^{\prime}} N(v)\right| \geq k_{2}$ holds, or it enumerates all subsets $V_{2}^{\prime} \subseteq V_{2}$ of size $k_{2}$ and checks whether $\left|\bigcap_{v \in V_{2}^{\prime}} N(v)\right| \geq k_{1}$ holds. If this is the case we clearly have found a $\left(k_{1}, k_{2}\right)$ biclique. This step can be done in time $\mathcal{O}^{*}\left(\min \left\{\left(\begin{array}{c}n_{1} \\ k_{1}\end{array}\right) ;\left(\begin{array}{c}n_{2} \\ k_{2}\end{array}\right)\right\}\right)$. By Vandermonde's identity, we have that for any $x, y, z \in \mathbb{N},\left(\begin{array}{c}x+y \\ z\end{array}\right)=\sum_{i=0}^{z}\left(\begin{array}{c}x \\ i\end{array}\right)\left(\begin{array}{c}y \\ z-i\end{array}\right)$. In particular, $\left(\begin{array}{l}n \\ k\end{array}\right)=\left(\begin{array}{l}n_{1}+n_{2} \\ k_{1}+k_{2}\end{array}\right) \geq\left(\begin{array}{l}n_{1} \\ k_{1}\end{array}\right) \cdot\left(\begin{array}{l}n_{2} \\ k_{2}\end{array}\right)$. Thus, $\min \left\{\left(\begin{array}{c}n_{1} \\ k_{1}\end{array}\right) ;\left(\begin{array}{l}n_{2} \\ k_{2}\end{array}\right)\right\} \leq \sqrt{\left(\begin{array}{c}n \\ k\end{array}\right)}$ and the running time of this step is upper bounded by $\mathcal{O}^{*}\left(\left(\begin{array}{c}n / 2 \\ a n / 2\end{array}\right)\right)=\mathcal{O}\left(1.30052^{n}\right)$.

This shows that Bipartite $\left(\mathbf{k}_{\mathbf{1}}, \mathbf{k}_{\mathbf{2}}\right)$ Biclique can be solved in $\mathcal{O}^{*}\left(1.30052^{n}\right)$ time. By Lemma 1, Constraint Bipartite Vertex Cover can also be solved in $\mathcal{O}^{*}\left(1.30052^{n}\right)$ time.

Corollary 3. Non-Induced $\left(\mathbf{k}_{\mathbf{1}}, \mathbf{k}_{\mathbf{2}}\right)$ Biclique can be solved in time $\mathcal{O}\left(1.6914^{n}\right)$, using polynomial space.

Proof. Given a graph $G=(V, E)$, construct a bipartite graph $G^{\prime}=\left(V, V^{\prime}, E^{\prime}\right)$ with $U^{\prime}=\left\{v^{\prime} \mid v \in U\right\}$ for $U \subseteq V$ and $\left\{u, v^{\prime}\right\} \in E^{\prime}$ iff $\{u, v\} \in E$. Observe that $(X, Y)$ is a non-induced $\left(k_{1}, k_{2}\right)$ biclique in $G$ if and only if $\left(X, Y^{\prime}\right)$ is a bipartite $\left(k_{1}, k_{2}\right)$ biclique in $G^{\prime}$. Now apply the preceding theorem to obtain a running time of $\mathcal{O}\left(1.30052^{2 n}\right)=$ $\mathcal{O}\left(1.6914^{n}\right)$. 


\section{Conclusions}

We already mentioned that CBVC, the parameterized dual of Non-induced $\left(\mathbf{k}_{\mathbf{1}}, \mathbf{k}_{\mathbf{2}}\right)$ Biclique, is in FPT. It is a natural question to ask whether Non-induced $\left(\mathbf{k}_{\mathbf{1}}, \mathbf{k}_{\mathbf{2}}\right)$ Biclique is in FPT as well. The proof of Kuo and Fuchs [12] (showing the NP-hardness of CBVC) is not parameter-preserving and hence does not answer this question. The only thing that can be (relatively easily) seen is membership in W[1]. As already mentioned in $[4,9,13]$, this poses an interesting open problem in parameterized complexity, even when restricted to bipartite graphs as in our sketched application and even when $k_{1}=k_{2}$. We finally mention that the experiments with an implementation of CBVC described in [2] show that the approach described in this paper might be feasible for many practical situations.

\section{References}

[1] J. Amilhastre, M.-C. Vilarem, and P. Janssen. Complexity of minimum biclique cover and minimum biclique decomposition for bipartite domino-free graphs. Discrete Applied Mathematics, 86:125-144, 1998.

[2] G. Bai and H. Fernau. Constraint bipartite vertex cover: Simpler exact algorithms and implementations. In F. P. Preparata, X. Wu, and J. Yin, editors, Frontiers in Algorithmics FAW, volume 5059 of $L N C S$, pages $67-78$. Springer, 2008.

[3] M. Dawande, P. Keskinocak, J. M. Swaminathan, and S. Tayur. On bipartite and multipartite clique problems. Journal of Algorithms, 41:388-403, 2001.

[4] E. Demaine, G. Z. Gutin, D. Marx, and U. Stege. 07281 Open problems. In Structure Theory and FPT Algorithmics for Graphs, Digraphs and Hypergraphs, number 07281 in Dagstuhl Seminar Proceedings, Dagstuhl, Germany, 2007. IBFI.

[5] V. M. Dias, C. M. de Figueiredo, and J. L. Szwarcfiter. Generating bicliques of a graph in lexicographic order. Theoretical Computer Science, 337(1-3):240-248, 2005.

[6] H. Fernau, S. Gaspers, D. Kratsch, M. Liedloff, and D. Raible. Exact exponential-time algorithms for finding bicliques in a graph. In S. Cafieri, A. Mucherino, G. Nannicini, F. Tarissan, and L. Liberti, editors, Cologne-Twente Workshop on Graphs and Combinatorial Optimization CTW, pages 205209, 2009.

[7] H. Fernau and R. Niedermeier. An efficient exact algorithm for constraint bipartite vertex cover. Journal of Algorithms, 38(2):374-410, 2001.

[8] M. R. Garey and D. S. Johnson. Computers and Intractability: A Guide to the Theory of NPCompleteness. Freeman, 1979.

[9] S. Gaspers. Exponential Time Algorithms: Structures, Measures, and Bounds. PhD thesis, Department of Informatics, University of Bergen, 2008.

[10] S. Gaspers, D. Kratsch, and M. Liedloff. On independent sets and bicliques in graphs. In H. Broersma, T. Erlebach, T. Friedetzky, and D. Paulusma, editors, Graph-Theoretic Concepts in Computer Science $W G$, volume 5344 of $L N C S$, pages 171-182, 2008.

[11] D. Hochbaum. Approximating clique and biclique problems. Journal of Algorithms, 29:174-200, 1998.

[12] S.-Y. Kuo and W. K. Fuchs. Efficient spare allocation for reconfigurable arrays. IEEE Design and Test, 4:24-31, Feb. 1987.

[13] D. Lokshtanov. New Methods in Parameterized Algorithms and Complexity. PhD thesis, Department of Informatics, University of Bergen, 2009.

[14] R. Peeters. The maximum edge biclique problem is NP-complete. Discrete Applied Mathematics, 131:651-654, 2003

[15] M. Yannakakis. Node and edge-deletion NP-complete problems. In Proc. 10th Annual ACM Symposium on Theory of Computing STOC, pages 253-264, 1978. 